\title{
Weibel instability in a plasma with nonzero external magnetic field
}

\author{
O. A. Pokhotelov and M. A. Balikhin
}

Automatic Control and Systems Engineering, University of Sheffield, Sheffield, UK

Correspondence to: O. A. Pokhotelov (o.a.pokhotelov@sheffield.ac.uk)

Received: 2 June 2012 - Revised: 17 June 2012 - Accepted: 18 June 2012 - Published: 13 July 2012

\begin{abstract}
The theory of the Weibel instability is generalized for the case of a plasma immersed in a nonzero external magnetic field. It is shown that the presence of this external field modifies the dispersion relation for this mode which now possesses a nonzero frequency. The explicit expression for the real and imaginary parts of the frequency is then calculated. It turns out that the linear growth rate remains unchanged, whereas the frequency becomes nonzero due to the finite value of the electron cyclotron frequency. The frequency of the Weibel mode is found to be proportional to the electron temperature anisotropy. The formal similarity of the Weibel and drift-mirror instabilities is outlined.
\end{abstract}

Keywords. Ionosphere (Plasma waves and instabilities)

\section{Introduction}

The Weibel instability (Weibel, 1959) is one of the most wellknown plasma instabilities. It arises in homogeneous plasmas possessing an electron velocity space anisotropy. The anisotropy can be explicitly described in terms of its perpendicular and parallel velocities, taking a bi-Maxwellian form. A simplified version of this instability has been considered by Fried (1959) who pointed out that it can be found in a plasma with the superposition of counter-streaming beams. In this respect it becomes similar to the ordinary two-stream instability (Schlickeiser and Shukla, 2003). The instability gives rise to the exponential growth of electromagnetic fields which help to restore plasma isotropy and is often considered as one of the most important mechanisms for the generation of magnetic fields in space (Medvedev and Loeb, 1999; Achtenberg and Wiersma, 2007). When a plasma is either free from or possesses negligibly small external magnetic fields, the Weibel wave behaves as a purely zero-frequency non propagating mode. However, in most plasma configurations, the system is not completely free of magnetic fields, some noticeable small external component is always present. The role of the seed perturbations, containing weak random magnetic fields, in the generation of Weibel instability has been clarified by Yoon (2007). Treumann and Baumjohann (2012) recently discussed the problem of the Weibel infrared catastrophe which arises due to the fact that magnetic field fluctuations are strongly dependent on their wave numbers $\left(\left\langle\delta B^{2}\right\rangle_{k} \propto k^{-3}\right)$. These authors also confirmed that thermal fluctuations can support a weak, spontaneous magnetic fluctuation background level in plasma and recalculated the thermal level for Weibel instability.

The aim of the present paper is to address another issue: to understand whether the Weibel instability can operate in a weak but steady external magnetic field and to determine the effect on the dispersion relation of the waves.

The paper is organized in the following fashion: in Sect. 2 we formulate the basic equations that describe the Weibel instability in a nonzero external magnetic field. The corresponding wave dispersion relation is derived in Sect. 3. In Sect. 3 the similarity between Weibel and drift-mirror instabilities is also pointed out. These results are then discussed in Sect. 4.

\section{Basic equations}

We consider a collisionless, uniform plasma composed of ions and electrons immersed in a nonzero external magnetic field $\boldsymbol{B}_{0}$. For the sake of simplicity we select the electron velocity distribution function to be bi-Maxwellian, whereas the ion distribution is represented by an ordinary Maxwellian. The components of the perturbed magnetic field $\delta \boldsymbol{B}$ take the form

$$
\delta B_{\mathrm{x}}=b_{\mathrm{x}}(t) e^{-i \omega+i k z}, \quad \delta B_{\mathrm{y}}=b_{\mathrm{y}}(t) e^{-i \omega+i k z}
$$


and

$\delta B_{\mathrm{Z}}=0$

We assume that all perturbed values vary as $\exp (-i \omega t+i k z)$ for each mode $(k, \omega), b_{\mathrm{x}, \mathrm{y}}(t)$ represents the magnetic field amplitudes. Furthermore, $\omega$ and $k$ are the wave frequency and wave number, respectively. We use a local Cartesian coordinate system in which the $\mathrm{z}$-axis is directed along the external magnetic field $\boldsymbol{B}_{0}$, and the $\mathrm{x}$ - and $\mathrm{y}$-axes are in the perpendicular plane, completing the triad. Our analysis will be limited to the most significant case, i.e. when the electron temperature is much greater than the ion temperature.

The phase velocity of the Weibel mode is much greater than the ion thermal speed. As a result the ions do not contribute to the Weibel dispersion relation. They simply act as a fixed neutralizing background. The linear response of the electrons to Weibel-type perturbations can be determined from the Vlasov equation

$$
\begin{aligned}
& -i\left(\omega-k v_{\|}\right) \delta f_{\omega, k}+\omega_{c} \frac{\partial \delta f_{\omega, k}}{\partial \alpha} \\
& =\frac{e b v_{\perp}}{m k}\left[\left(\omega-k v_{\|}\right) \frac{\partial F}{v_{\perp} \partial v_{\perp}}+k \frac{\partial F}{\partial v_{\|}}\right] \exp (\mp i \alpha) .
\end{aligned}
$$

We consider that for an undisturbed velocity distribution function $F$ that is symmetric between $v_{\mathrm{x}}$ and $v_{\mathrm{y}}$, the moduli of the components of the magnetic field $b_{\mathrm{x}}$ and $b_{\mathrm{y}}$ are equal, i.e. $\left|b_{\mathrm{x}}\right|=\left|b_{\mathrm{y}}\right|$. We may further assume that $b_{\mathrm{x}}= \pm i b_{\mathrm{y}} \equiv$ $\pm i b$. We note that $b_{\mathrm{x}}= \pm b_{\mathrm{y}}$ corresponds to a rotation of the system of reference in the $(\mathrm{x}, \mathrm{y})$ plane. The components $v_{\mathrm{x}}$ and $v_{\mathrm{y}}$ are related to $v_{\perp}$ and pitch-angle $\alpha$ through the relations $v_{\mathrm{x}}=v_{\perp} \cos \alpha$ and $v_{\mathrm{y}}=v_{\perp} \sin \alpha$ and $v_{\|}$is the component of the particle velocity along the external magnetic field. Furthermore, $m$ is the electron mass, $e$ is the magnitude of the electron charge and $\omega_{\mathrm{c}}=e B_{0} / m$ is the electron cyclotron frequency.

Searching for a solution to Eq. (2) in the form $\delta f_{\omega, k}=$ $\delta f_{\omega, k}^{\mp} \exp (\mp i \alpha)$ one finds

$$
\begin{aligned}
\delta f_{\omega, k}^{\mp}= & i \frac{e b v_{\perp}}{m k}\left[\frac{\partial F}{v_{\perp} \partial v_{\perp}}-\frac{\partial F}{v_{\|} \partial v_{\|}}\right. \\
& \left.+\frac{\omega \frac{\partial F}{v_{\|} \partial v_{\|}} \pm \omega_{\mathrm{c}}\left(\frac{\partial F}{v_{\perp} \partial v_{\perp}}-\frac{\partial F}{v_{\|} \partial v_{\|}}\right)}{\omega-k v_{\|} \pm \omega_{\mathrm{c}}}\right] .
\end{aligned}
$$

Here the \pm sign corresponds to the clock-wise (right-hand) or anti-clockwise (left-hand) rotation of the wave polarization plane.

\section{Weibel dispersion relation}

Using the distribution function (3), we find the $\mathrm{x}$-component of the electric current

$$
\begin{aligned}
j_{\mathrm{X}}= & -i \frac{e^{2} b \pi}{2 m k} \int v_{\perp}^{3} d v_{\perp} d v_{\|}\left[\frac{\partial F}{v_{\perp} \partial v_{\perp}}-\frac{\partial F}{v_{\|} \partial v_{\|}}\right. \\
& \left.+\frac{\omega \frac{\partial F}{v_{\|} \partial v_{\|}} \pm \omega_{\mathrm{c}}\left(\frac{\partial F}{v_{\perp} \partial v_{\perp}}-\frac{\partial F}{v_{\|} \partial v_{\|}}\right)}{\omega-k v_{\|} \pm \omega_{\mathrm{c}}}\right] .
\end{aligned}
$$

Substituting Eq. (4) into Ampére's law and neglecting the contribution of the displacement current, one finds that the wave dispersion relation takes the form:

$$
\begin{aligned}
& \frac{k^{2} c^{2}}{\omega_{p}^{2}}-\frac{\pi}{n} \int v_{\perp}^{3} d v_{\perp} d v_{\|}\left[\frac{\partial F}{v_{\perp} \partial v_{\perp}}-\frac{\partial F}{v_{\|} \partial v_{\|}}\right. \\
& \left.+\frac{\omega \frac{\partial F}{v_{\|} \partial v_{\|}} \pm \omega_{\mathrm{c}}\left(\frac{\partial F}{v_{\perp} \partial v_{\perp}}-\frac{\partial F}{v_{\|} \partial v_{\|}}\right)}{\omega-k v_{\|} \pm \omega_{\mathrm{c}}}\right]=0,
\end{aligned}
$$

where $c$ is the velocity of light, $n$ is the plasma number density, $\omega_{p}=\left(n e^{2} / m \varepsilon_{0}\right)^{1 / 2}$ is the Langmuir frequency and $\varepsilon_{0}$ is the permittivity of free space. The integration is carried out over velocity space.

Integrating Eq. (5) over $v_{\perp}$ by parts we have

$$
\begin{aligned}
& I-1-\frac{k^{2} c^{2}}{\omega_{p}^{2}}+\frac{\pi}{n} \int v_{\perp}^{3} d v_{\perp} d v_{\|} \\
& \times \frac{\omega \frac{\partial F}{v_{\|} \partial v_{\|}} \pm \omega_{\mathrm{c}}\left(\frac{\partial F}{v_{\perp} \partial v_{\perp}}-\frac{\partial F}{v_{\|} \partial v_{\|}}\right)}{\omega-k v_{\|} \pm \omega_{\mathrm{c}}}=0,
\end{aligned}
$$

where

$$
I=-\frac{\pi}{n} \int v_{\perp}^{3} d v_{\perp} d v_{\|} \frac{\partial F}{v_{\|} \partial v_{\|}} .
$$

Formally, the dispersion relation (6) is somewhat similar to that for the drift-mirror mode (cf. Hasegawa, 1969 (Eq. 36); Pokhotelov et al., 1985 (Eq. 46)). A comparison between these expressions shows that in the case of the Weibel instability, the role of the finite ion Larmor radius effect is played by the collisionless skin depth $c / \omega_{p}$, the role of the mirror force is played by the first two terms in the square brackets in Eq. (5) and the role of the diamagnetic drift frequency is replaced by the electron cyclotron frequency $\omega_{\mathrm{c}}$.

Let us now analyze the dispersion relation (6) for the case when the equilibrium electron velocity distribution function takes a bi-Maxwellian form given by

$F=\frac{n}{\pi^{3 / 2} v_{T_{\perp}}^{2} v_{T_{\|}}} \exp \left(-\frac{v_{\perp}^{2}}{v_{T_{\perp}}^{2}}-\frac{v_{\|}^{2}}{v_{T_{\|}}^{2}}\right)$,

where $v_{T_{\perp(\mid)}}=\left(2 T_{\perp(||)} / m\right)^{1 / 2}$ is the perpendicular (parallel) electron thermal velocity and $T_{\perp(\|)}$ is the electron perpendicular (parallel) electron temperature. Other investigations 
have dealt with more exotic anisotropic distributions, such as waterbag (Yoon and Davidson, 1987) or kappa-distributions (Zaheer and Murtaza, 2007). However, the use of the distribution (8) results in a standard form for the dispersion relation which is useful for various applications. Substituting Eq. (8) into Eq. (6) and making necessary integrations one finds

$$
\begin{aligned}
\frac{T_{\perp}}{T_{\|}}-1 & -\frac{k^{2} c^{2}}{\omega_{p}} \\
& +\frac{T_{\perp}}{T_{\|}} \frac{\omega \mp \omega_{\mathrm{c}}\left(T_{||} / T_{\perp}-1\right)}{|k| v_{T_{\|}}} Z\left(\frac{\omega \pm \omega_{\mathrm{c}}}{|k| v_{T_{\|}}}\right)=0,
\end{aligned}
$$

where $Z(\zeta)$ is the plasma dispersion function given by

$$
Z(\zeta)=\frac{1}{\pi^{\frac{1}{2}}} \int_{-\infty}^{\infty} \frac{e^{-y^{2}} d y}{y-\zeta}
$$

The resonant Weibel instability is found in the low-frequency limit $\left|\omega \pm \omega_{\mathrm{c}}\right| \ll\left|k v_{\|}\right|$, where the following expansion holds

$$
Z(\zeta) \simeq i \pi^{1 / 2}-2 \zeta
$$

Substituting Eq. (11) into Eq. (9) and decomposing the frequency into its imaginary and real parts one finds (cf. Pokhotelov and Amariutei, 2011)

$\operatorname{Im}(\omega)=\frac{|k| v_{T_{\|}}}{\pi^{1 / 2}} \frac{T_{\|}}{T_{\perp}}\left(\frac{T_{\perp}}{T_{\|}}-1-\frac{k^{2} c^{2}}{\omega_{p}^{2}}\right)$,

and

$\operatorname{Re}(\omega)= \pm \omega_{\mathrm{c}} \frac{T_{\|}}{T_{\perp}}\left[\left(1-\frac{2}{\pi}\right)\left(\frac{T_{\perp}}{T_{\|}}-1\right)+\frac{2}{\pi} \frac{k^{2} c^{2}}{\omega_{p}^{2}}\right]$.

One sees that the instability leads to the excitation of two circularly polarized waves moving in the opposite directions along the magnetic field. The maximum growth rate occurs when

$k=k_{\max }=\frac{\omega_{p}}{3^{1 / 2} c}\left(\frac{T_{\perp}}{T_{\|}}-1\right)^{\frac{1}{2}}$.

Substituting Eq. (14) into Eq. (13) one finds that the frequency of the fastest growing mode is

$(\operatorname{Re} \omega)_{\max }= \pm 0.58 \times \omega_{c}\left(1-T_{\|} / T_{\perp}\right) \ll \omega_{\mathrm{c}}$.

Thus, under conditions of marginal stability, which were assumed in the derivation presented here, the Weibel mode remains low frequency even in the presence of nonzero external magnetic field.

\section{Discussion and conclusions}

In the present study we have investigated the effect of a nonzero external magnetic field in relation to the Weibel instability. It was shown that the inclusion of this field leads to the following modifications. First, when the magnetic field $B_{0}$ is much smaller than $\left(T_{\perp} / T_{||}-1\right)^{1 / 2}\left(\mu_{0} p_{\perp}\right)^{1 / 2}$ (where $\mu_{0}$ is permeability of free space and $p_{\perp}$ is the plasma pressure), the Weibel instability growth rate remains unchanged. It takes the form (12), which coincides with the classical expression (e.g. Weibel, 1959; see also Pokhotelov and Amariutei, 2011). One would expect that in stronger, not really strong, fields not only the frequency will increase coming closer to the electron cyclotron frequency, but also changes in threshold and decrease in growth rate will result. In case of Weibel two-stream instability, the corresponding analysis was carried out by Hededal and Nishikawa (2005) using PIC simulations. In this study the external magnetic field reduces the instability growth rate. However, such an analysis we reserve for our future investigations. Second, in the presence of an external magnetic field, the Weibel mode has nonzero frequency defined by Eq. (13) or Eq. (15) in contrast to the case of zero external magnetic field. The instability generates two circularly polarized modes moving in the opposite directions along the external magnetic field.

One of the interesting features of the Weibel mode in the presence of nonzero external magnetic field is that Eq. (6) is mathematically similar to that derived by Hasegawa (1969) for the drift-mirror mode. The nonlinear theory of the Weibel instability including a nonzero external magnetic field can be constructed in a similar way to that carried out by Pokhotelov et al. (2010) and Pokhotelov and Amariutei (2011).

Acknowledgements. This research was supported by STFC, by FP7 (grant no. 262005) and by RFBR (grant no. 11-05-00920). Authors are grateful to ISSI staff for support.

Topical Editor R. Nakamura thanks two anonymous referees for their help in evaluating this paper.

\section{References}

Achtenberg, A. and Wiersma, J.: The Weibel instability in relativistic plasmas. I. Linear theory, II. Nonlinear theory and stabilization mechanism, Astron. Astrophys. 475, 1-18, doi:10.1051/0004-6361:20065365, 2007.

Fried, B. D.: Mechanism for instability of transverse plasma waves, Phys. Fluids, 2, 337-337, 1959.

Hasegawa, A.: Drift mirror instability in the magnetosphere, Phys. Fluids, 12, 2642-2650, 1969.

Hededal, C. B. and Nishikawa, K.-I.: The influence of an ambient magnetic field on relativistic collisionless plasma shocks, Astrophys. J., 623, L89-L92, 2005.

Medvedev, M. V. and Loeb, A.: Generation of magnetic fields in the relativistic shock of gamma-ray burst sources, Astrophys. J., 526, 697-706, doi:10.1086/308038, 1999. 
Pokhotelov, O. A. and Amariutei, O. A.: Quasi-linear dynamics of Weibel instability, Ann. Geophys., 29, 1997-2001, doi:10.5194/angeo-29-1997-2011, 2011.

Pokhotelov O. A., Pilipenko, V. A., and Amata, E.: Drift anisotropy instability of a finite $\beta$ magnetospheric plasma, Planet. Space Sci., 33, 1229-1241, 1985.

Pokhotelov, O. A., Sagdeev, R. Z., Balikhin, M. A., Fedun, V. N., and Dudnikova, G. I.: Nonlinear Mirror and Weibel modes: peculiarities of quasi-linear dynamics, Ann. Geophys., 28, 21612167, doi:10.5194/angeo-28-2161-2010, 2010.

Schlickeiser, R. and Shukla, P. K.: Cosmological magnetic field generation by the Weibel instability, Astrophys. J. Lett., 599, L57-L60, doi:10.1086/381246, 2003.
Treumann, R. A. and Baumjohann, W.: A note on the Weibel instability and thermal fluctuations, Ann. Geophys., 30, 427-431, doi:10.5194/angeo-30-427-2012, 2012.

Weibel, E. S.: Spontaneously growing transverse waves in a plasma due to an anisotropic velocity distribution, Phys. Rev. Lett., 2, 83-84, 1959.

Yoon, P. H.: Spontaneous thermal magnetic field fluctuations, Phys. Plasmas, 14, 064504-064504-4, doi:10.1063/1.2741388, 2007.

Yoon, P. H. and Davidson, R. C.: Exact analytical model of the classical Weibel instability in a relativistic anisotropic plasma, Phys. Rev. A, 35, 2718-2721, doi:10.1103/PhysRevA.35.2718, 1987.

Zaheer, S. and Murtaza, G.: Weibel instability with non-Maxwellian distribution functions, Phys. Plasmas, 14, 022108, 2007. 